\title{
Low-Intensity Shockwave Therapy (LI-ESWT) in Diabetic Kidney Disease: Results from an Open-Label Interventional Clinical Trial
}

Sune Moeller Skov-Jeppesen

Knud Bonnet Yderstraede

Boye $L$ Jensen

Claus Bistrup (D)

Milad Hanna

Lars Lund

'Department of Urology, Odense University Hospital, Odense, Denmark; ${ }^{2}$ OPEN, Odense Patient data Explorative Network, Odense University Hospital, Odense, Denmark; ${ }^{3}$ Clinical Institute, University of Southern Denmark, Odense, Denmark; ${ }^{4}$ Department of Endocrinology, Odense University Hospital, Odense, Denmark; ${ }^{5}$ Department of Cardiovascular and Renal Research, Institute of Molecular Medicine, University of Southern Denmark, Odense, Denmark; ${ }^{6}$ Department of Nephrology, Odense University Hospital, Odense, Denmark; ${ }^{7}$ Department of Urology, Charing Cross Hospital, Imperial College Healthcare NHS Trust, London, UK
Correspondence: Sune Moeller Skov-Jeppesen

Department of Urology, Odense

University Hospital, Sdr. Boulevard 29,

Odense C, 5000, Denmark

$\mathrm{Tel}+4551210911$

Fax +45654II726

Email sun_mjep@hotmail.com
Purpose: Treatment with low-intensity shockwave therapy (LI-ESWT) is associated with angiogenesis and is suggested as a treatment for different types of vascular diseases. It was hypothesized that LI-ESWT improves the renal filtration barrier and halts the progression of GFR decline in diabetic kidney disease (DKD) potentially through VEGF and NO formation. We present the first data on LI-ESWT in human DKD.

Methods: The study was designed as an interventional, prospective, one-arm, Phase 1 study. We investigated change in GFR and albuminuria in 28 patients with DKD treated with six sessions of LI-ESWT over three weeks. The patients were followed for six months. Urine excretion of kidney injury markers, vascular endothelial growth factor (VEGF) and nitric oxide metabolites (NOx) was studied after LI-ESWT.

Results: There were no significant changes in GFR and albuminuria up to six months after LI-ESWT compared to baseline. Urine VEGF was transiently reduced one month after LIESWT, but there were no other significant changes in urine VEGF or NOx after LI-ESWT. Secondary analysis showed that NOx increased after LI-ESWT in patients who had low levels of NOx at baseline. Kidney injury marker trefoil factor 3 (TFF3) increased acutely after the first session of LI-ESWT indicating transient endothelial repair. Other markers of kidney injury were stable in relation to LI-ESWT.

Conclusion: LI-ESWT treatment did not significantly improve kidney function and albumin excretion. It is concluded that LI-ESWT is not harmful. A randomized blinded study should be performed to clarify whether adjunctive treatment with LI-ESWT is superior to standard treatment of DKD.

Keywords: albuminuria, clinical trial, diabetic kidney disease, glomerular filtration rate, ESWT, extracorporeal shockwave therapy

\section{Introduction}

Diabetes mellitus (DM) affect 493 million adult individuals worldwide ${ }^{1}$ and is associated with a number of complications reducing the life-span and quality of life of patients living with $\mathrm{DM}^{2}$ One of the most serious and devastating complications of DM is the presence of diabetic kidney disease (DKD) which may ultimately lead to end-stage kidney disease and considerably increased risk of death. DKD is estimated to affect $20-40 \%$ of all patients with $\mathrm{DM}^{3}$ with increasing prevalence found in older patients. Thus, more than $50 \%$ of patients with DM aged $>65$ years might be affected by DKD. ${ }^{4}$

We recently proposed low-intensity shockwave therapy (LI-ESWT) as a new renoprotective intervention to reduce the progression of DKD. LI-ESWT is applied with 
approximately $10 \%$ of the energy level that is used in the setting of lithotripsy (ESWL). LI-ESWT can be applied to different organs exposed to ischemia in order to induce angiogenesis via upregulation of local vascular endothelial growth factor (VEGF) and nitric oxide (NO) production. ${ }^{5-7}$ Other potential beneficial effects associated with LI-ESWT include attraction of stem cells ${ }^{8,9}$ and reduction of fibrosis $^{10,11}$ in target organs. Clinically, LI-ESWT reduces symptoms in ischemic heart disease ${ }^{12}$ and peripheral arterial disease. ${ }^{13}$ Furthermore, LI-ESWT improves the healing of diabetic foot ulcers ${ }^{14}$ and was recently recommended for vasculogenic erectile dysfunction. ${ }^{15}$

LI-ESWT is a type of acoustic wave characterized by a high peak positive pressure followed by a slightly negative, tensile pressure that causes shear stress of the cell membrane, cytoskeleton, and extracellular matrix components. ${ }^{16,17}$ The application of LI-ESWT leads to activation of different intracellular signaling pathways involved in endothelial cell proliferation and angiogenesis by stimulation of specific cell membrane receptor proteins ${ }^{18,19}$ and cell membrane poration. ${ }^{20}$ LI-ESWT can affect tissue fibrosis and inflammation through a suppression of TGF-beta release, ${ }^{21}$ NF-kappa-beta pathway, ${ }^{22}$ and interleukins 1 and $6^{23}$ leading to decreased infiltration of fibrocytes, ${ }^{10}$ leukocytes, ${ }^{24}$ and inflammatory macrophages. ${ }^{23}$

The findings that LI-ESWT may suppress inflammation and fibrosis and promote regeneration in vascular diseases motivated the hypothesis that LI-ESWT alleviates the microvascular complications associated with DKD ie, reduced GFR and albuminuria. To test the hypothesis, a protocol with six interventions over a period of three weeks was performed on 28 patients with DKD. The patients were followed for six months.

We previously reported preliminary data demonstrating the clinical safety of LI-ESWT in a subset of the cohort of patients with DKD. ${ }^{25}$ The present study reports the full set of data after completing the trial with six-month follow-up on all included patients. The effect of LI-ESWT treatment on glomerular filtration rate (GFR), albuminuria, VEGF and NO excretion, blood pressure, and kidney injury markers is shown in the full cohort of patients with DKD.

\section{Patients and Methods}

The study was designed as a one-arm, prospective, interventional cohort study. We recruited 28 patients with diabetic kidney disease between May 27th, 2015 and June 27th, 2019 at Odense University Hospital, Odense, Denmark. The inclusion and exclusion criteria for the study are presented
Box I Inclusion and Exclusion Criteria

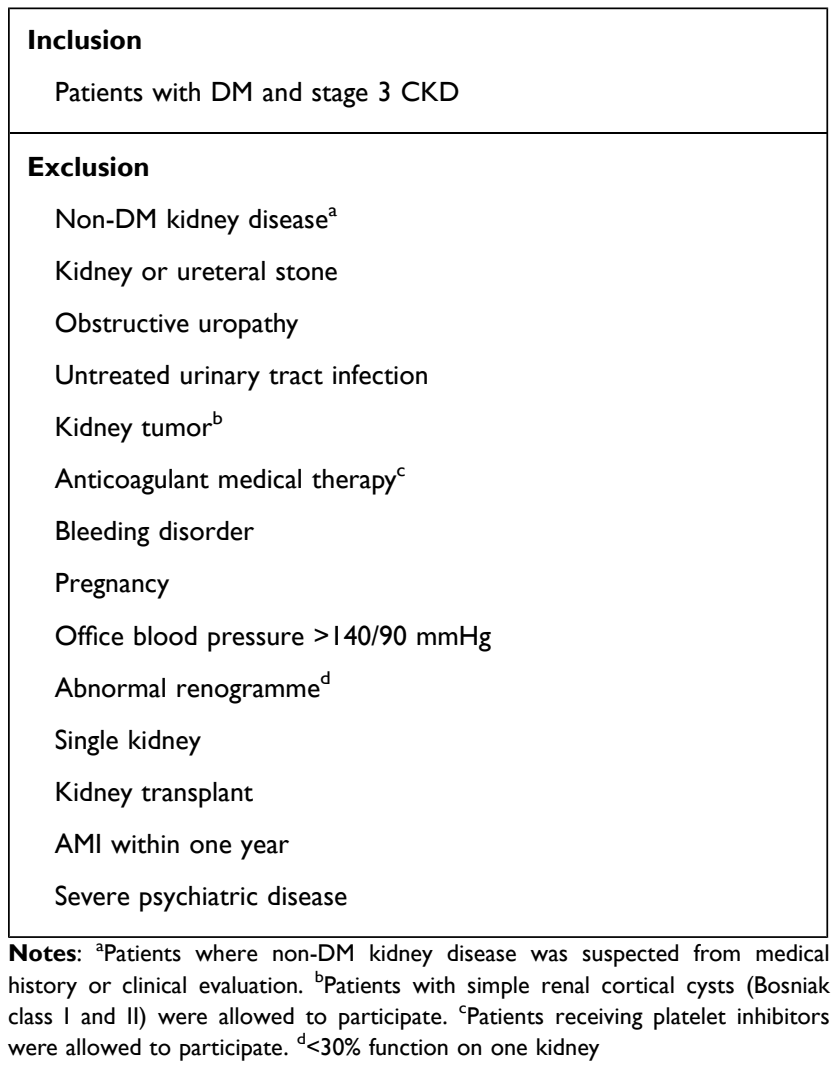

in Box 1. Patients received treatment with Li-ESWT and were followed up at one month, three months, and six months after the last treatment session with LI-ESWT. An overview of study investigations is provided in Figure 1.

Preliminary results from the present trial were published in a safety report. ${ }^{25}$ In the present analysis, the results were reported for a total of 28 patients. Only 14 patients were included in our preliminary safety report.

\section{Li-ESWT}

Six treatment sessions with LI-ESWT were carried out over a period of three weeks. There were three to four days interval between each of the treatment sessions. The treatment was carried out using a Modulith SLX-2 device (Storz Medical, Tägerwilen, Switzerland). At every session, each kidney was treated with 1000 shocks at the upper pole, 1000 shocks at the middle part, and 1000 shocks at the lower pole. The energy level was increased stepwise at every part of the kidney within the first 200 shocks; we started at minimum energy setting at $0.1(0.136$ $\mathrm{mJ} / \mathrm{mm} 2$ ) and gradually increased up to energy setting 0.7 $(0.265 \mathrm{~mJ} / \mathrm{mm} 2)$. The remaining 800 shock at each part of the kidney were applied with energy setting 0.7. All 


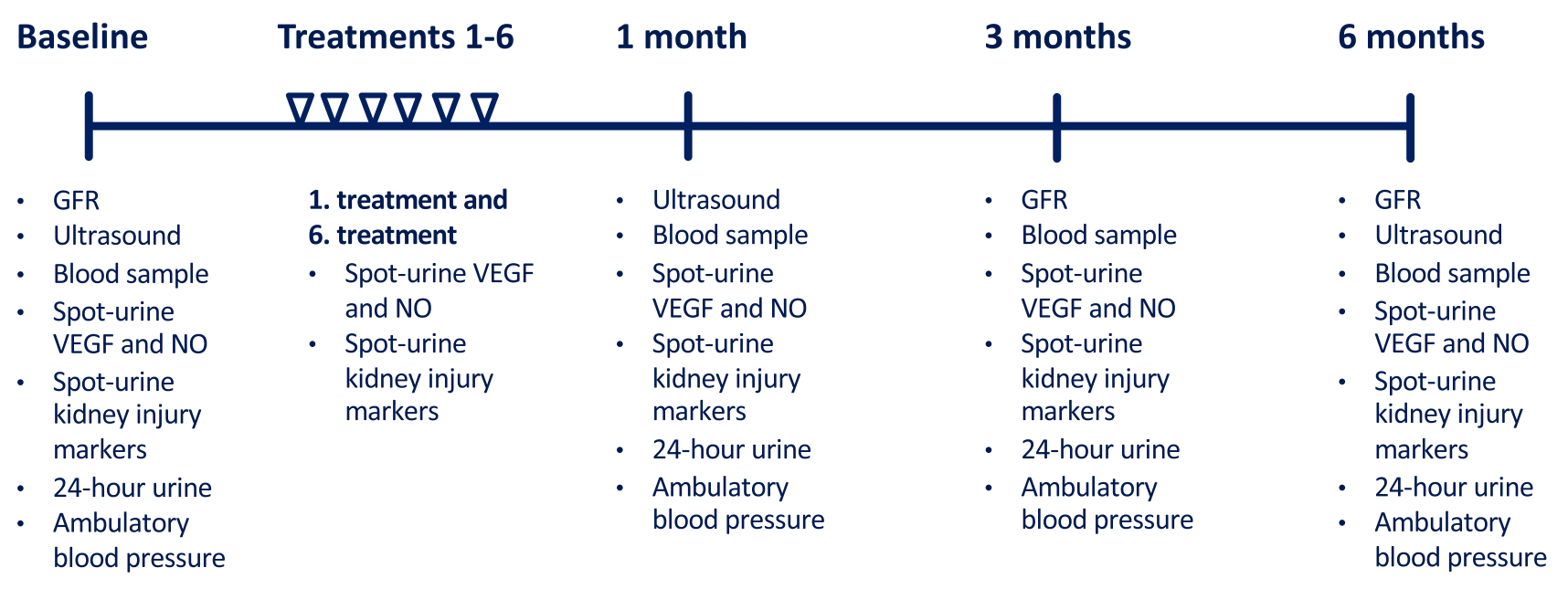

Figure I Overview of investigations.

shocks were applied with extended focal size and $4 \mathrm{~Hz}$ frequency. The patients were placed on their back or side during the treatments. We used water as coupling medium between the device and the patients' skin. The kidneys were localized in-line with ultrasound.

\section{Outcomes}

GFR was determined with 51-chromium-EDTA or Tc-99mDTPA clearance method. Albuminuria was quantified in 24hour urine collection samples. We measured ambulatory blood pressure using an Ambulatory Blood Pressure Monitor model 90217A (Spacelabs Healthcare, USA) with blood pressure readings every 20 minutes during daytime from 6 am to 10 pm and every 60 minutes during the nighttime. Blood samples were drawn from a peripheral vein. Concise methods for these outcomes were previously described. ${ }^{25}$

\section{Spot Urine Samples}

Nitric oxide metabolites (NOx), VEGF and kidney injury markers were measured in spot urine samples taken at baseline, at $1 \mathrm{st}$ and 6 th treatment sessions, and at followup after 1, 3, and 6 months. At the 1st treatment session, the spot urine sample was taken immediately after LIESWT to assess the acute effects of the treatment. At the 6th treatment session, the spot urine sample was taken before LI-ESWT to assess the subacute effects of the previous treatment which was conducted 3-4 days earlier.

The concentrations of VEGF and kidney injury markers calbindin, clusterin, kidney injury molecule-1 (KIM-1), osteoactivin, and trefoil factor 3 (TFF3) were determined in spot urine samples with a commercially available multiplex ELISA assay (Kidney Injury Panel 3 Human Kit, Meso
Scale Diagnostics, Rockville, Maryland, USA). Nitric oxide metabolites nitrite and nitrate (NOx) were quantified in spot urine samples using a commercial colorimetric assay (Nitrate/Nitrite Colorimetric assay Kit, Cayman Chemical, Ann Arbor, Michigan, USA).

The concentrations of the analytes were determined in duplicate and normalized to creatinine concentration.

\section{Statistics}

Non-parametric Friedman test with level of significance 0.05 was used for GFR, albuminuria, ambulatory blood pressure, and plasma variables. Unadjusted post-hoc test was carried out for significant outcomes of the Friedman test. We excluded six patients with non-significant excretion of albumin in urine $(<30 \mathrm{mg} / 24$-hours through all study visits) from the analysis of albuminuria. Levels of NOx, VEGF, and kidney injury markers were compared to baseline with Wilcoxon signed rank test with significance level set to 0.10 in order to prevent from type II errors evaluating the safety of LI-ESWT.

We performed an exploratory analysis of correlations between the variables obtained at baseline, treatment sessions, and follow-up. Linear correlations between continuous variables were tested using Pearson's product-moment correlation. Non-linear correlations between continuous variables were tested using Spearman's rank-order correlation.

All statistics were performed in SPSS Statistics version 26 for Mac (IBM, Armonk, NY, USA).

\section{Results}

Twenty-eight included patients completed the treatments with LI-ESWT as per protocol. One patient dropped out of the 
study after a three-month follow-up visit. All other patients completed the follow-up program and study investigations. There were twenty male patients and eight female patients (Table 1). The patients had a median age of 61 years (IQR 54; 69) and were diagnosed with type $2 \mathrm{DM}(\mathrm{n}=19)$, type $1 \mathrm{DM}$ $(\mathrm{n}=6)$, latent autoimmune diabetes in adults (LADA) $(\mathrm{n}=2)$, or maturity onset diabetes of the young type 3 (MODY3) $(n=1)$. The median duration of DM was 25 years (IQR 13; 35).

\section{Clinical Safety}

A mild degree of macroscopic hematuria was observed after the treatment with LI-ESWT in six of the patients but was not associated with significant adverse renal outcomes. Macroscopic hematuria was only present in the patients' first urine after the treatments and was dissolved at their next void. Microscopic hematuria was observed in six patients (excluding the patients who had macroscopic hematuria) after the treatments. In general, the patients experienced transient mild to moderate flank tenderness lasting two to three days after the treatments with LI-ESWT. All of the patients reported that flank tenderness was reduced before a new treatment session was initiated and did not increase from time to time through the treatments. Ultrasound scans performed one and three months after LI-ESWT showed no hematoma in the kidneys.

\section{Functional Outcomes}

There were no significant changes in the patients' GFR and albuminuria after LI-ESWT (Table 3). At baseline, median GFR was $37.0 \mathrm{~mL} / \mathrm{min} / 1.73 \mathrm{~m} 2$ (IQR 27.3; 49.0) compared to $36.0 \mathrm{~mL} / \mathrm{min} / 1.73 \mathrm{~m} 2$ (IQR $27.0 ; 46.8$ ) at three-month follow-up and $37.0 \mathrm{~mL} / \mathrm{min} / 1.73 \mathrm{~m} 2$ (IQR $24.0 ; 51.0$ ) at sixmonth follow-up $(\mathrm{p}=0.351)$. Median urinary albumin excretion was $302 \mathrm{mg} / 24$-hours (IQR 78; 786) at baseline, $253 \mathrm{mg} /$ 24-hours (IQR 80; 1187) at one-month follow-up, $295 \mathrm{mg} /$ hours $(58 ; 907)$ at three-month follow-up, and $214 \mathrm{mg}$ /hours (100; 987) at six-month follow-up $(\mathrm{p}=0.801)$. Individual curves representing GFR and albuminuria in each patient during the study are provided in Figure 2.

Sodium excretion, potassium excretion, or volume in 24-hour urine samples did not change significantly after LI-ESWT. Median creatinine clearance was $56.2 \mathrm{~mL} / \mathrm{min}$ (IQR 36.5; 70.1) at baseline and was not significantly affected by LI-ESWT $(\mathrm{p}=0.165)$.

Median baseline systolic ambulatory blood pressure was $126 \mathrm{mmHg}$ (IQR 119; 133) and median baseline diastolic ambulatory blood pressure was $70 \mathrm{mmHg}$ (IQR 64; 79). The ambulatory blood pressure did not change through follow-up visits compared to baseline.
Table I Baseline Demographics

\begin{tabular}{|c|c|}
\hline \multicolumn{2}{|l|}{ Sex } \\
\hline Male & $20(7 \mid \%)$ \\
\hline Female & $8(29 \%)$ \\
\hline Age (years) & $6 I(54 ; 69)$ \\
\hline Body mass index $(\mathrm{kg} / \mathrm{m} 2)$ & $28(22 ; 32)$ \\
\hline \multicolumn{2}{|l|}{ Smoking } \\
\hline Current & $3(11 \%)$ \\
\hline Former & 15 (54\%) \\
\hline Never & 10 (36\%) \\
\hline Package years, none-smokers excluded & $18(7 ; 4 \mid)$ \\
\hline Alcohol consumption (12g/week) & $\mathrm{I}(0 ; 5)$ \\
\hline \multicolumn{2}{|l|}{ Diabetes mellitus } \\
\hline Type I & $6(21 \%)$ \\
\hline Type 2 & $19(68 \%)$ \\
\hline Other (MODY/LADA) & $3(11 \%)$ \\
\hline Duration of diabetes (years) & $25(13 ; 35)$ \\
\hline \multicolumn{2}{|l|}{ Medical treatment of diabetes mellitus } \\
\hline None & I (4\%) \\
\hline Metformin & $7(25 \%)$ \\
\hline Insulin & $23(82 \%)$ \\
\hline Sulfunylurea & $2(7 \%)$ \\
\hline DPP-4 inh./GLP-I-RA/SGLT2-inh. & $7(25 \%)$ \\
\hline \multicolumn{2}{|l|}{ Medical treatment of hypertension } \\
\hline None & $3(11 \%)$ \\
\hline ACE-inhibitor /AT2-antagonist & $23(82 \%)$ \\
\hline Thiazide & $14(50 \%)$ \\
\hline Furosemide & $12(43 \%)$ \\
\hline Beta-blocker & $8(29 \%)$ \\
\hline Calcium-antagonist & $15(54 \%)$ \\
\hline Other & $9(32 \%)$ \\
\hline Receiving lipid-lowering medication & $25(89 \%)$ \\
\hline \multicolumn{2}{|l|}{ Charlson Index score ${ }^{a}$} \\
\hline 2 & $12(43 \%)$ \\
\hline $3-4$ & $13(46 \%)$ \\
\hline 5 or more & 3 (II\%) \\
\hline
\end{tabular}

Notes: Data are number (\%) or median (25\% quartile ; $75 \%$ quartile). ${ }^{a}$ Minimum Charlson Index Score is 2 according to the diagnosis of diabetic nephropathy in all patients.

Adjustments made to the patients' anti-hypertensive and anti-diabetic medical treatment during follow-up are outlined in Appendix 1.

\section{Plasma Variables}

There were no significant changes in hemoglobin, albumin, sodium, potassium, ionized calcium, phosphate, creatinine, or glycated hemoglobin throughout the study 
A

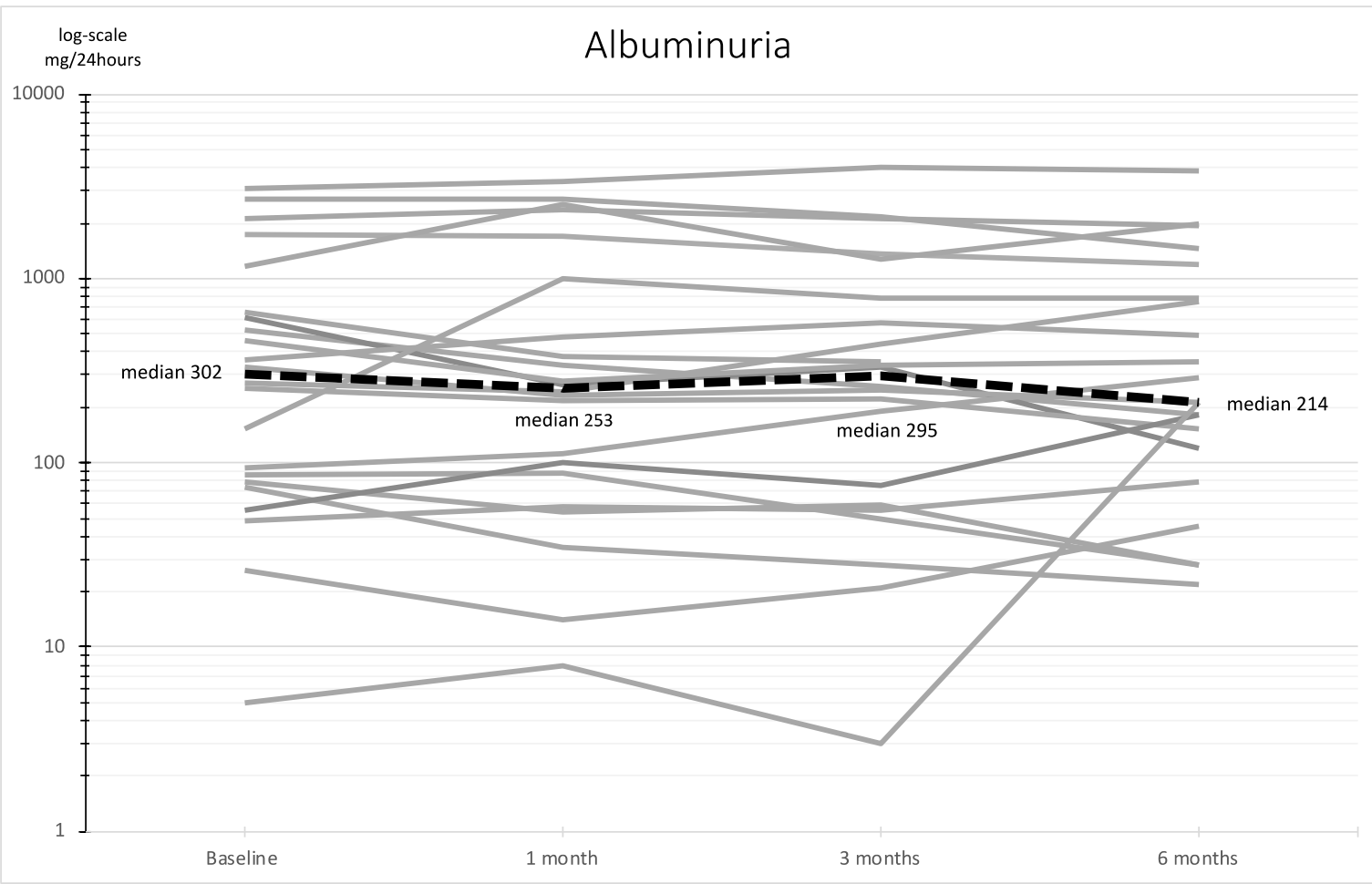

\section{B}

GFR

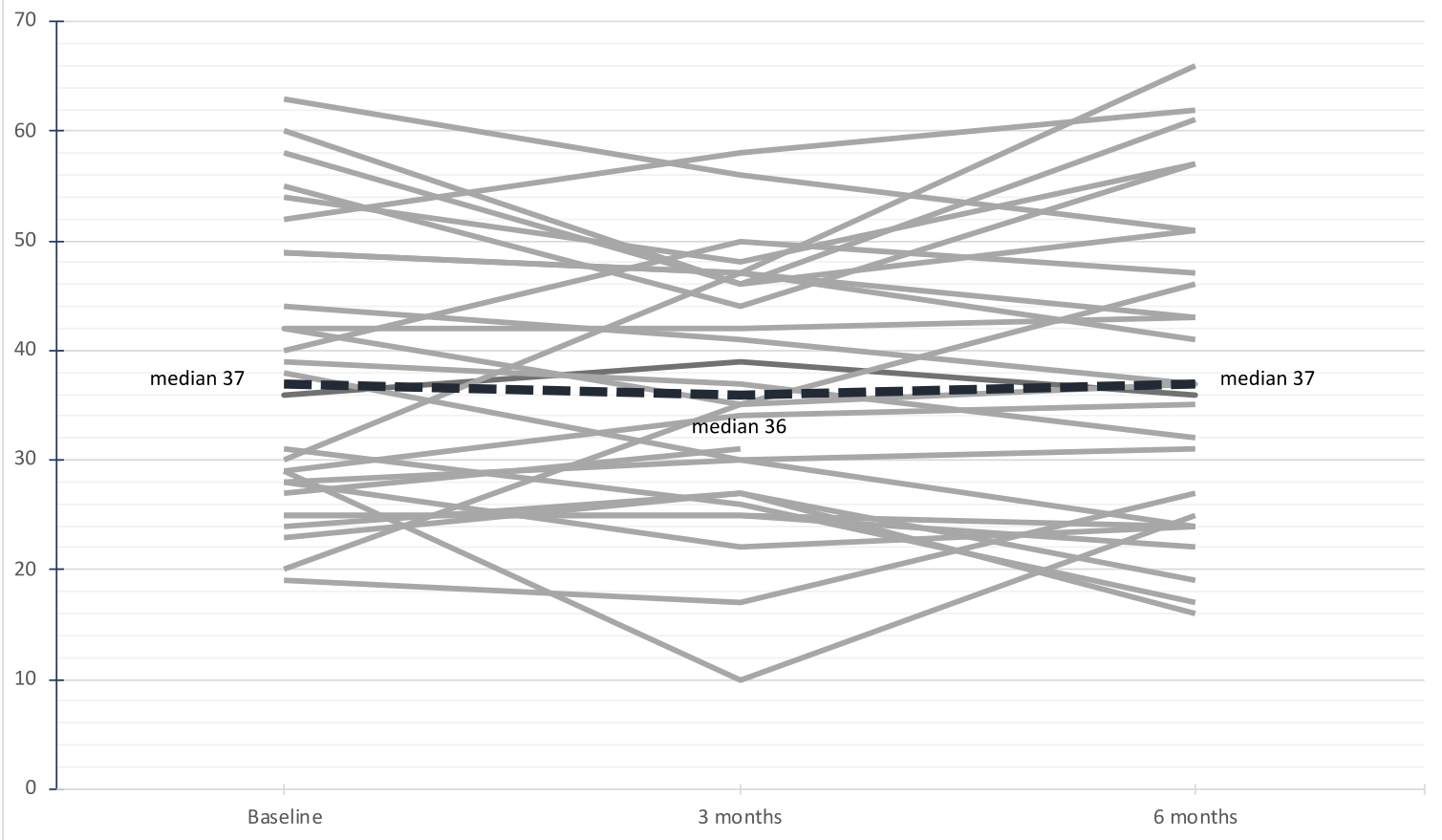

Figure 2 Individual curves representing albuminuria (A) and GFR (B) in each patient during the study. 
(Table 2). There was a significant increase in BUN $(\mathrm{p}=0.038)$. At baseline, median BUN was $10.4 \mathrm{mmol} / \mathrm{L}$ (IQR $8.8 ; 14.3$ ) and increased at six-month follow-up visit where the median was $13.7 \mathrm{mmol} / \mathrm{L}$ (IQR 8.6; 15.8). In a post-hoc test, there was a significant increase in BUN from baseline to six-month follow-up visit $(p=0.018)$ and from three-month follow-up visit to six-month follow-up visit $(\mathrm{p}=0.031)$.

\section{NOx and VEGF Excretion}

In spot urine samples, the concentration of NOx concentration increased numerically after LI-ESWT but not in a statistically significant way (Table 3). Median urine VEGF was $76.0 \mathrm{ng} / \mathrm{mmol}(36.3 ; 123.1)$ at baseline and decreased significantly to $54.9 \mathrm{ng} / \mathrm{mmol}(39.5 ; 101.7)$ at one-month follow-up $(p=0.056)$. There were no further significant differences in urine VEGF excretion. Individual curves representing NOx and VEGF in each patient during the study are provided in Figure 3.

\section{Markers of Kidney Injury}

Median urine TFF3 increased significantly to $23.1 \mathrm{ng} /$ mmol $(12.2 ; 66.5)$ after the first treatment session compared to a baseline median value of $13.3 \mathrm{ng} / \mathrm{mmol}(8.6$; 33.7) $(p=0.030)$ (Table 3). However, at all other visits, there were no significant changes in TFF3 relative to baseline. KIM-1 and clusterin tended to decrease after the first treatment session compared to baseline but the results were not statistically significant. After the first treatment, median urine KIM-1 was $69.8 \mathrm{ng} / \mathrm{mmol}(47.0 ; 120.7)$ compared to $394 \mathrm{ng} / \mathrm{mmol}(62.2 ; 1150)$ at baseline $(\mathrm{p}>0.10)$. Median urine clusterin was $4699 \mathrm{ng} / \mathrm{mmol}$ $(2780 ; 17,732)$ after the first treatment in comparison to $8137 \mathrm{ng} / \mathrm{mmol}(3131 ; 23,468)$ at baseline $(\mathrm{p}>0.10)$. Individual curves representing TFF3 in each patient during the study are provided in Figure 3.

\section{Correlation Analyses Between Outcomes}

There were significant correlations between the recorded variables with an overview provided in Appendix 2. Notably, the levels of NOx, calbindin, clusterin, KIM-1, osteoactivin, TFF3, and VEGF tended to change after LIESWT in a manner that depended on the baseline level. The kidney injury markers decreased in those patients that displayed high baseline level of these markers. The patients who had a low baseline level of injury markers tended to have an increase in these markers after LIESWT. NOx and VEGF tended to increase in the patients who had low baseline levels of NOx and VEGF but were reduced in the patients with high levels at baseline. These effects were evident in close relation to the treatment sessions ie first treatment, sixth treatment, and 1-month follow-up. However, the effect persisted at six-month follow-up for NOx and calbindin. Furthermore, after the first treatment session, the change in VEGF correlated positively with the change in NOx.

\section{Discussion}

The present study found no significant changes in GFR and albuminuria after LI-ESWT. Albuminuria decreased in half of the patients after LI-ESWT but with no overall significant decline. BUN increased at six-month follow-up compared to baseline but was stable after one month and three months. Plasma concentrations of creatinine, potassium, and phosphate and creatinine clearance were unaffected by LI-ESWT. In the present study, the side effects to LI-ESWT were comparable to our previous report. ${ }^{25}$ In summary, LI-ESWT is clinically and biochemically safe in human diabetic kidney disease.

To our knowledge, this study represents the first data on LI-ESWT in human kidney disease. A previous study in a pig model of kidney disease demonstrated that LIESWT improved renal microvascular density, tissue oxygenation, and GFR following ischemic kidney injury. ${ }^{26}$ Thus, LI-ESWT promoted a regenerative angiogenic response in kidneys with ischemic injury through a mechanism involving stimulation of focal adhesion kinase and beta 1-integrin that transmit the mechanical stimulus of LI-ESWT. ${ }^{26}$ In animals, VEGF increased after LI-ESWT in various types of renal disease ${ }^{26-28}$ including type-1 DKD. ${ }^{29}$ Furthermore, eNOS expression improved after LI-ESWT in animal models of acute ischemic renal disease ${ }^{26}$ and chronic renal disease. ${ }^{28}$ However, in hypertensive renal disease in rats, LI-ESWT did not improve VEGF or eNOS expression. ${ }^{30}$ Interestingly, recent animal data suggested that LI-ESWT increase the expression of stromal-derived cell factor-1 and promote homing of endothelial progenitor cells to injured renal tissue. ${ }^{31}$

In spot urine samples, we found no overall changes in NOx. VEGF was transiently reduced after one month. Previous studies show that NOx is increased immediately after shockwave lithotripsy (ESWL) in both plasma and urine. $^{32}$ In rodents, ESWL leads to activation of the NOcGMP pathway which may counteract renal vasoconstriction. $^{33}$ In particular, ESWL causes renal 


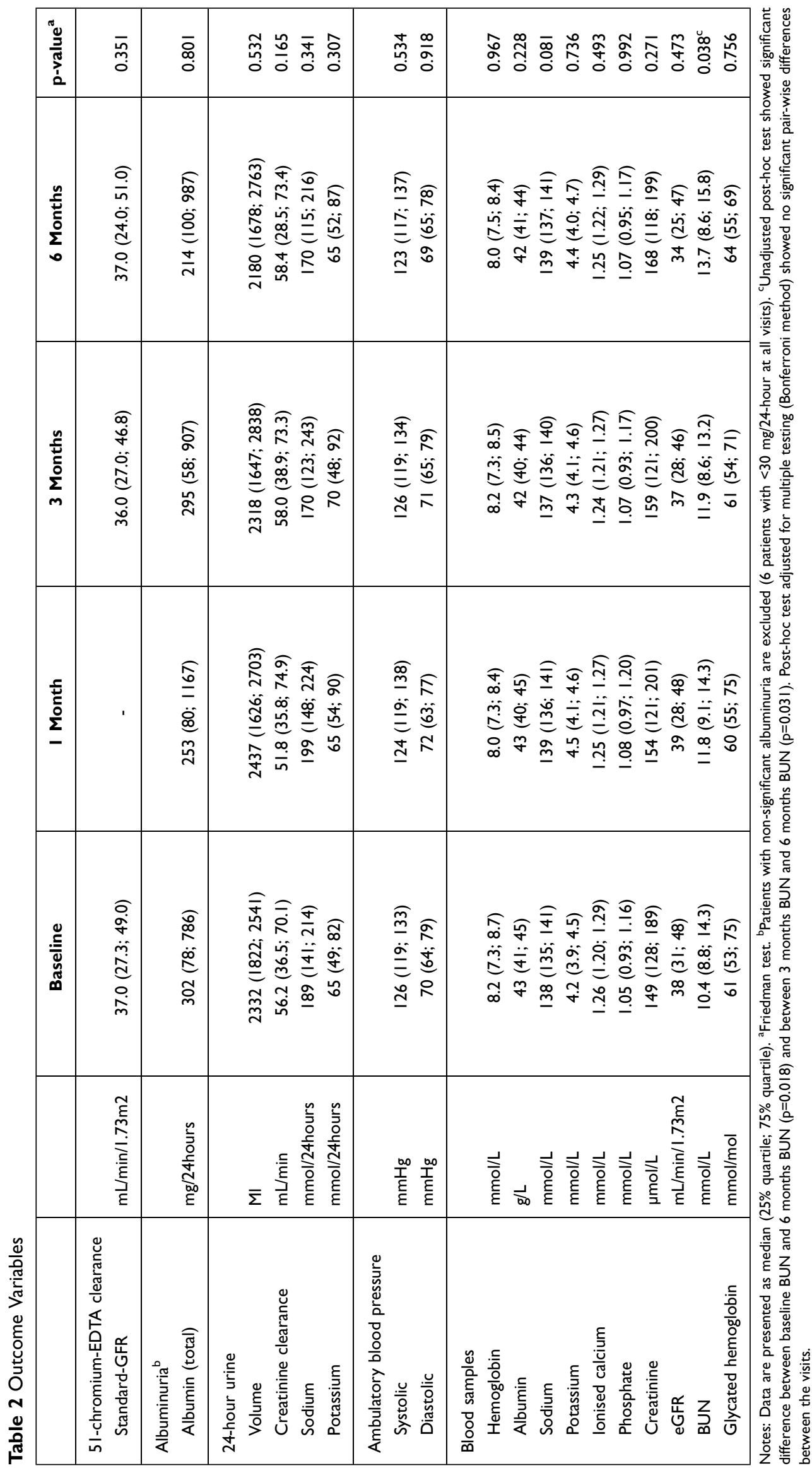




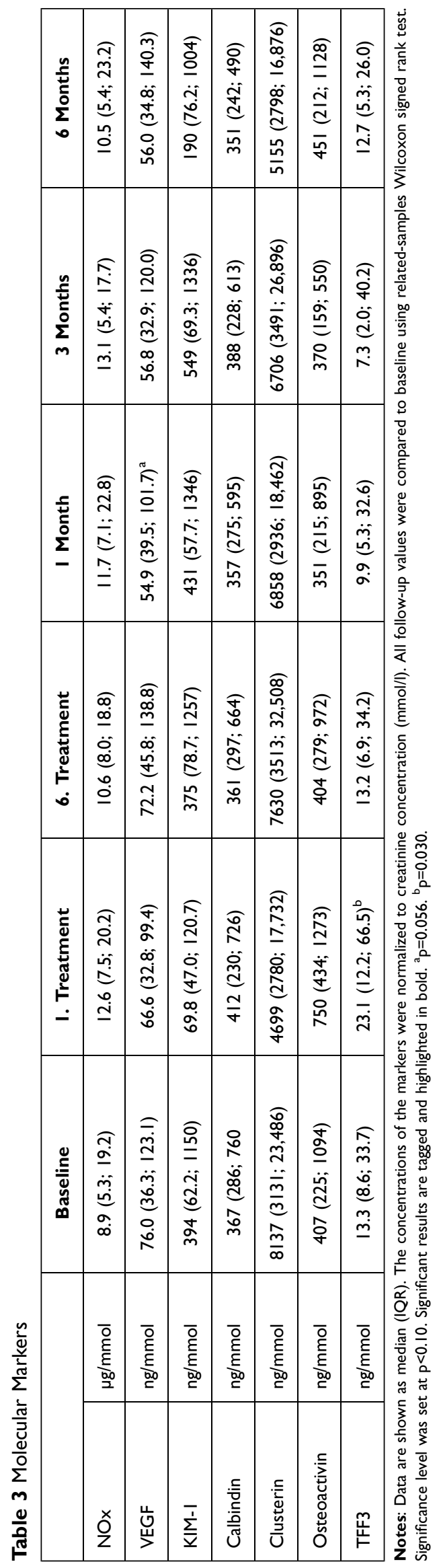

vasoconstriction in humans, ${ }^{34}$ and we therefore speculate that the production of NOx could be secondary to transient renal ischemia induced by shockwaves. In the present study, we did not measure renal blood flow or resistive index during LI-ESWT to further investigate this mechanism.

Kidney injury markers in spot urine were not significantly affected by LI-ESWT except TFF3 which increased at the first treatment session. TFF3 is a peptide that plays a protective role for epithelial surfaces in the gastrointestinal tract but its function in the kidney is not fully clarified. ${ }^{35}$ In rodents, TFF3 decreased in urine and kidney tissue a few days following tubular injury whereas urinary levels of albumin, KIM-1, and clusterin increased. ${ }^{36,37}$ Seemingly, TFF3 changes in an opposite pattern compared to other kidney injury markers after acute tubular injury. However, in human chronic kidney disease, urinary levels of TFF3 are increased suggesting on-going epithelial repair. ${ }^{38}$ In the present study, the increase in TFF3 was short lasting $(<$ two or three days after LI-ESWT since the level of TFF3 returned to baseline level in the spot urine sample that was obtained before initiation of the sixth treatment session) and could indicate transiently increased epithelial injury/repair as a response to LI-ESWT. Such interpretation would fit also with mild hematuria observed in some patients after LI-ESWT. Nonetheless, we found no correlation of the increase in TFF3 with changes in primary functional outcomes GFR and albuminuria.

In rats, LI-ESWT reduced serum levels of kidney injury markers neutrophil gelatinase-associated lipocalin (NGAL) and KIM-1 and renal tissue injury after renal reperfusion injury. ${ }^{39}$ In the present study, KIM-1 and clusterin decreased in the majority of patients after the first session of LI-ESWT but not overall statistically significantly. In animal studies, LI-ESWT reduced renal fibrosis following chronic proteinuric kidney disease ${ }^{28}$ and after renal ischemic injury or reperfusion injury. ${ }^{26,40} \mathrm{We}$ did not include renal tissue data or circulating fibrosis biomarkers in the present report to investigate the potential of LIESWT to reduce tissue fibrosis. Osteoactivin is suggested to trigger upregulation of collagen and matrix metalloproteinase expression and induce renal interstitial fibrosis. ${ }^{41}$ However, we found no change in the urinary excretion of osteoactivin after LI-ESWT.

In a secondary correlation analysis, we found a remarkable association between the urinary level of NOx, VEGF, and kidney injury markers at baseline and the respective changes in these molecules during 


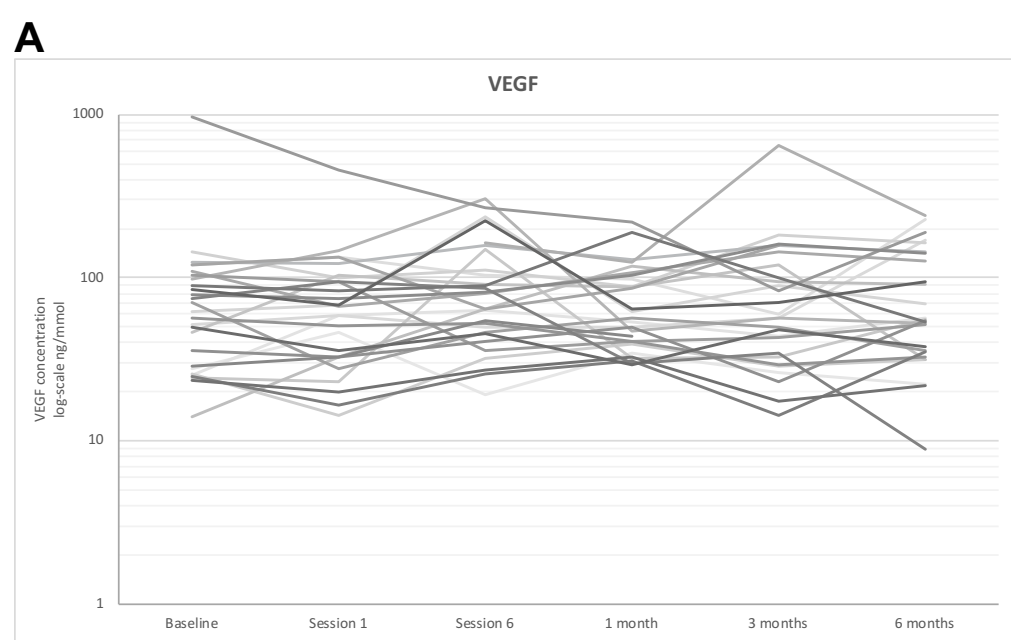

B

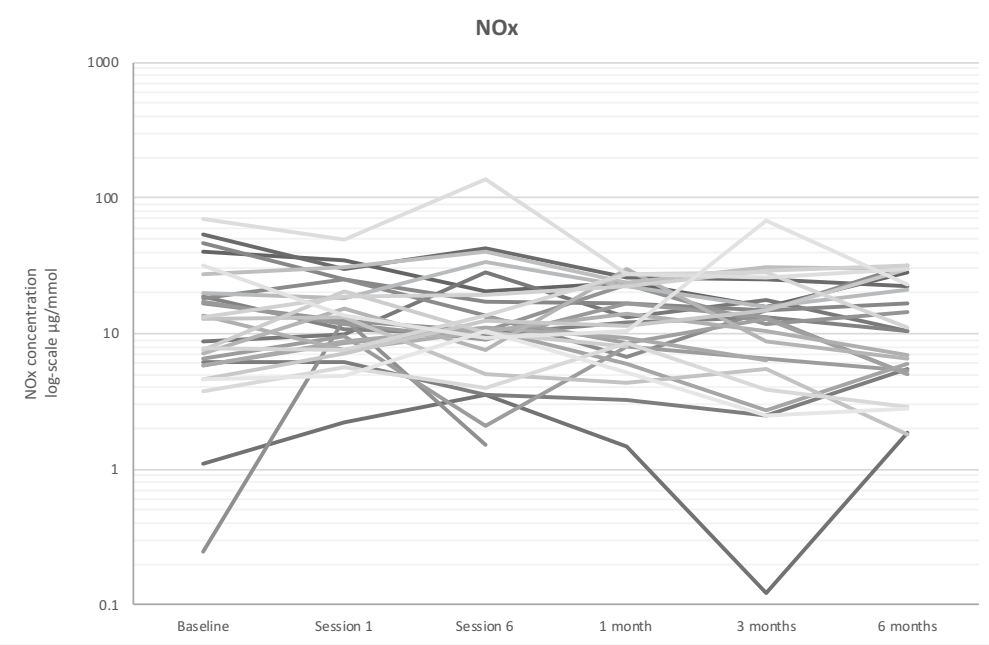

C

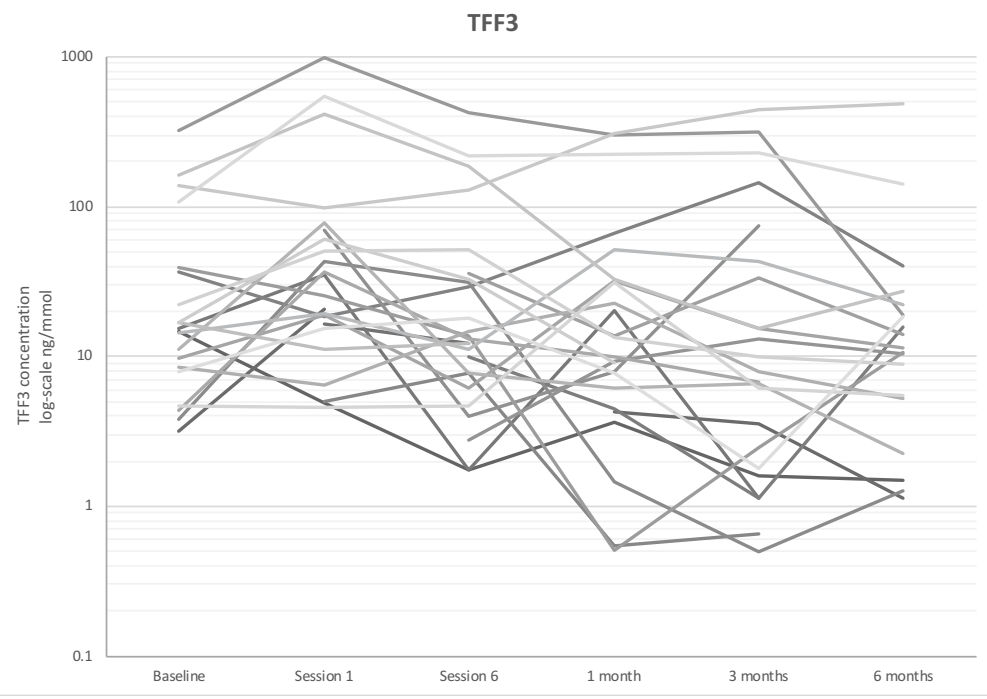

Figure 3 Individual curves representing VEGF (A), NOx (B) and TFF3 (C) in each patient during the study. 
treatments and follow-up. Thus, there was a tendency towards an increase in NOx in urine after LI-ESWT in those patients who had a relatively low excretion of NOx at baseline. Apparently, with increasing baseline levels, the changes in the NOx, VEGF, and kidney injury markers decreased linearly after LI-ESWT. That also meant that NOx and VEGF tended to decrease in the patients who had a relatively high level at baseline. Interpreting these results, the patients with most extreme excretions of NOx, VEGF, and kidney injury markers regressed towards mean levels after LI-ESWT. In the present study, we found no explanation as to why this effect in the kidney was seen.

At baseline, we found that albuminuria was present almost exclusively in patients with relatively low levels of NOx and relatively high levels of VEGF, and that lower NOx or higher VEGF was associated with exponentially increasing albuminuria. In animals, VEGF is increased under the circumstances of DKD. ${ }^{42}$ Conversely, in some studies of human DKD, VEGF is reduced and VEGF antagonism lead to adverse renal outcomes. ${ }^{43}$ It is suggested that in DKD there is an impairment of the VEGF-NO axis where VEGF is uncoupled from its normal stimulation of $\mathrm{NO}$ and regulation of angiogenesis. ${ }^{44}$ The fact that NOx tended to increase after LI-ESWT in the patients who had a low level of NOx at baseline could potentially work as a protective factor against albuminuria. In the patients who had increasing VEGF during the treatment sessions and early follow-up, GFR tended to improve later after three and six months. It is possible that VEGF may be used in future studies to divide patients in response/non-response groups after LI-ESWT. However, in correlation analysis, we found no other clinical feature or biomarker that could be used to predict changes in GFR, albuminuria, or blood pressure. After the first session of LIESWT, VEGF and NOx acutely changed in the same direction within the patients as demonstrated in our secondary correlation analysis. However, at follow-up, there was no clear relationship between the change in NOx and the change in VEGF that could indicate a possible restoration of the balance between VEGF and NO.

Our study is limited in that no control group was implemented. Furthermore, the patients were followed for relatively short time (six months) which, in the absence of LI-ESWT, may be too short to record significant changes in GFR or albuminuria in patients with diabetic kidney disease. We did not count how many patients were screened for participation. It is possible that our study population is selective considering that the most fragile or comorbid patients might have declined participation due to the relatively intense study program. We detected no significant changes in GFR and albuminuria after LI-ESWT demonstrating that LI-ESWT is not harmful. In perspective, LI-ESWT can prevent loss of kidney function or reduce the rate of kidney functional decline, but a control group and longer-term follow-up is needed to evaluate such effects. Moreover, the interventions should likely be repeated in order to maintain the effect on long-term. In patients with DKD, small annual reductions in GFR is expected in both type-1 $\mathrm{DM}^{45}$ and type-2 $\mathrm{DM}^{46}$ In the present study, we carefully monitored covariates that could bias GFR and albuminuria. There were no changes in ambulatory blood pressure or HbAlc during follow-up. Two patients discontinued their treatment with ACE-inhibitor early during follow-up possibly constituting a bias for renal functional outcomes. LI-ESWT elicited a number of positive effects in animal models of renal disease. ${ }^{26,28,29}$ However, in these studies, LI-ESWT was applied with lower energy at $0.09-0.13 \mathrm{~mJ} / \mathrm{mm} 2$ compared to our study. Future clinical studies need focus on doseresponse relationship of LI-ESWT. It is also likely that the effective dose of LI-ESWT energy received in the kidney might be variable based on different patients' weight and soft tissue thickness. In futures studies, it would be useful to determine whether there is a need to individually adjust the energy level of LI-ESWT based on factors such as skin to kidney distance.

In conclusion, we report that LI-ESWT is clinically safe and does not adversely affect markers of kidney injury in patients with DKD. We found no change in primary renal functional outcomes, ie GFR and albuminuria. LIESWT had no overall effect on VEGF and NOx excretion in urine. An exploratory analysis indicated that NOx increased in patients who had low levels of NOx. VEGF temporarily increased after LI-ESWT in patients with low level of VEGF. Furthermore, an increase in VEGF after LI-ESWT was associated with improving GFR. We recommend that LI-ESWT is evaluated in a randomized, placebo-controlled trial.

\section{Data Sharing Statement}

No data will be shared.

\section{Ethics Approval}

The study followed the Helsinki II declaration on clinical trials and was registered and approved at national authorities; the Regional Committees on Health Research Ethics for Southern Denmark (ID S-20130150) and the Danish Data Protection Agency (regional registration 2008-58- 
0035, journal no. 15/34140). The study was registered at ClinicalTrials.gov (ID NCT02515461).

\section{Consent to Participate}

All patients provided signed informed consent to participate.

\section{Author Contributions}

All authors contributed to data analysis, drafting or revising the article, have agreed on the journal to which the article will be submitted, gave final approval of the version to be published, and agree to be accountable for all aspects of the work.

\section{Funding}

Funding was received from the Beckett-Foundation and the A.P. Møller Foundation for the Advancement of Medical Science. No part of the study was influenced from funding organizations.

\section{Disclosure}

S.M.S.J. received a grant from Storz Medical AG during the conduct of the study and reported no other potential conflicts of interest for this work. M.H. is advisor to Storz Medical AG and has received non-financial support and personal fees from Storz Medical AG and reported no other potential conflicts of interest for this work. The remaining authors declare they have no conflicts of interest for this work. The results presented in this paper have not been published previously in whole or part.

\section{References}

1. Saeedi P, Petersohn I, Salpea P, et al. Global and regional diabetes prevalence estimates for 2019 and projections for 2030 and 2045: results from the International Diabetes Federation Diabetes Atlas, 9 (th) edition. Diabetes Res Clin Pract. 2019;157:107843. doi:10.1016/j. diabres.2019.107843

2. Green A, Sortso C, Jensen PB, Emneus M. Incidence, morbidity, mortality, and prevalence of diabetes in Denmark, 2000-2011: results from the Diabetes Impact Study 2013. Clin Epidemiol. 2015;7:421-430. doi:10.2147/CLEP.S88577

3. American Diabetes Association. Standards of medical care in diabetes--2014. Diabetes Care. 2014;37(Suppl 1):S14-80. doi:10.2337/dc14-S014

4. de Boer IH, Rue TC, Hall YN, Heagerty PJ, Weiss NS, Himmelfarb J. Temporal trends in the prevalence of diabetic kidney disease in the United States. JAMA. 2011;305(24):2532-2539. doi:10.1001/ jama.2011.861

5. Yan X, Zeng B, Chai Y, Luo C, Li X. Improvement of blood flow, expression of nitric oxide, and vascular endothelial growth factor by low-energy shockwave therapy in random-pattern skin flap model. Ann Plast Surg. 2008;61(6):646-653. doi:10.1097/SAP.0b 013e318172ba1f
6. Fu M, Sun CK, Lin YC, et al. Extracorporeal shock wave therapy reverses ischemia-related left ventricular dysfunction and remodeling: molecular-cellular and functional assessment. PLoS One. 2011;6 (9):e24342. doi:10.1371/journal.pone.0024342

7. Wang C-J, Ko J-Y, Kuo Y-R, Yang Y-J. Molecular changes in diabetic foot ulcers. Diabetes Res Clin Pract. 2011;94(1):105-110. doi:10.1016/j.diabres.2011.06.016

8. Aicher A, Heeschen C, Sasaki K, Urbich C, Zeiher AM, Dimmeler S. Low-energy shock wave for enhancing recruitment of endothelial progenitor cells: a new modality to increase efficacy of cell therapy in chronic hind limb ischemia. Circulation. 2006;114(25):2823-2830. doi:10.1161/CIRCULATIONAHA.106.628623

9. Gollmann-Tepekoylu C, Lobenwein D, Theurl M, et al. Shock wave therapy improves cardiac function in a model of chronic ischemic heart failure: evidence for a mechanism involving VEGF signaling and the extracellular matrix. $J$ Am Heart Assoc. 2018;7(20):e010025. doi:10.1161/JAHA.118.010025

10. Lei PP, Tao SM, Shuai Q, et al. Extracorporeal cardiac shock wave therapy ameliorates myocardial fibrosis by decreasing the amount of fibrocytes after acute myocardial infarction in pigs. Coron Artery Dis. 2013;24(6):509-515. doi:10.1097/MCA.0b013e3283640ec7

11. Assaly R, Giuliano F, Clement P, et al. Extracorporeal shock waves therapy delivered by aries improves erectile dysfunction in spontaneously hypertensive rats through penile tissue remodeling and neovascularization. Sex Med. 2019;7(4):441-450. doi:10.1016/j. esxm.2019.08.006

12. Yang P, Guo T, Wang W, et al. Randomized and double-blind controlled clinical trial of extracorporeal cardiac shock wave therapy for coronary heart disease. Heart Vessels. 2013;28(3):284-291. doi:10.1007/s00380-012-0244-7

13. Cayton T, Harwood A, Smith GE, Chetter I. A systematic review of extracorporeal shockwave therapy as a novel treatment for intermittent claudication. Ann Vasc Surg. 2016;35:226-233. doi:10.1016/j. avsg.2016.02.017

14. Snyder R, Galiano R, Mayer P, Rogers LC, Alvarez O. Diabetic foot ulcer treatment with focused shockwave therapy: two multicentre, prospective, controlled, double-blinded, randomised Phase III clinical trials. J Wound Care. 2018;27(12):822-836. doi:10.12968/jowc.2018.27.12.822

15. EAU Guidelines. Edn. Presented at the EAU Annual Congress Amsterdam 2020. Arnhem, The Netherlands: EAU Guidelines Office; 2020. ISBN 978-94-92671-07-3.

16. d'Agostino MC, Craig K, Tibalt E, Respizzi S. Shock wave as biological therapeutic tool: from mechanical stimulation to recovery and healing, through mechanotransduction. Int J Surg. 2015;24 (PtB):147-153. doi:10.1016/j.ijsu.2015.11.030

17. Gerdesmeyer L, Maier M, Haake M, Schmitz C. Physikalisch-technische Grundlagen der extrakorporalen Stoßwellentherapie (ESWT) [Physical-technical principles of extracorporeal shockwave therapy (ESWT)]. Orthopade. 2002;31(7):610-617. German. doi:10.1007/ s00132-002-0319-8

18. Ha CH, Kim S, Chung J, An SH, Kwon K. Extracorporeal shock wave stimulates expression of the angiogenic genes via mechanosensory complex in endothelial cells: mimetic effect of fluid shear stress in endothelial cells. Int $J$ Cardiol. 2013;168(4):4168-4177. doi:10.1016/j.ijcard.2013.07.112

19. Hatanaka K, Ito K, Shindo T, et al. Molecular mechanisms of the angiogenic effects of low-energy shock wave therapy: roles of mechanotransduction. Am J Physiol Cell Physiol. 2016;311(3): C378-85. doi:10.1152/ajpcell.00152.2016

20. Holfeld J, Tepekoylu C, Reissig C, et al. Toll-like receptor 3 signalling mediates angiogenic response upon shock wave treatment of ischaemic muscle. Cardiovasc Res. 2016;109(2):331-343. doi:10.1093/cvr/cvv272

21. Sheu JJ, Ali HEE, Cheng BC, et al. Extracorporeal shock wave treatment attenuated left ventricular dysfunction and remodeling in mini-pig with cardiorenal syndrome. Oncotarget. 2017;8 (33):54747-54763. doi:10.18632/oncotarget.18287 
22. Mariotto S, Cavalieri E, Amelio E, et al. Extracorporeal shock waves: from lithotripsy to anti-inflammatory action by NO production. Nitric Oxide. 2005;12(2):89-96. doi:10.1016/j.niox.2004.12.005

23. Abe Y, Ito K, Hao K, et al. Extracorporeal low-energy shock-wave therapy exerts anti-inflammatory effects in a rat model of acute myocardial infarction. Circ J. 2014;78(12):2915-2925. doi:10.1253/ circj.CJ-14-0230

24. Kuo YR, Wang CT, Wang FS, Chiang YC, Wang CJ. Extracorporeal shock-wave therapy enhanced wound healing via increasing topical blood perfusion and tissue regeneration in a rat model of STZ-induced diabetes. Wound Repair Regen. 2009;17(4):522-530. doi:10.1111/j.1524-475X.2009.00504.x

25. Skov-Jeppesen SM, Yderstraede KB, Bistrup C, et al. Low-intensity shockwave therapy in the treatment of diabetic nephropathy: a prospective Phase 1 study. Nephrol Dial Transplant. 2018.

26. Zhang X, Krier JD, Amador Carrascal C, et al. Low-energy shockwave therapy improves ischemic kidney microcirculation. J Am Soc Nephrol. 2016;27(12):3715-3724. doi:10.1681/ASN.2015060704

27. Yoshida M, Nakamichi T, Mori T, Ito K, Shimokawa H, Ito S. Lowenergy extracorporeal shock wave ameliorates ischemic acute kidney injury in rats. Clin Exp Nephrol. 2019;23(5):597-605. doi:10.1007/ s10157-019-01689-7

28. Sung PH, Chen KH, Li YC, Chiang JY, Lee MS, Yip HK. Sitagliptin and shock wave-supported peripheral blood derived endothelial progenitor cell therapy effectively preserves residual renal function in chronic kidney disease in rat-role of dipeptidyl peptidase 4 inhibition. Biomed Pharmacother. 2019;111:1088-1102. doi:10.1016/j. biopha.2019.01.025

29. Hsiao CC, Huang WH, Cheng KH, Lee CT. Low-energy extracorporeal shock wave therapy ameliorates kidney function in diabetic nephropathy. Oxid Med Cell Longev. 2019;2019:8259645. doi:10.1155/2019/8259645

30. Caron J, Michel PA, Dussaule JC, Chatziantoniou C, Ronco P, Boffa JJ. Extracorporeal shock wave therapy does not improve hypertensive nephropathy. Physiol Rep. 2016;4(11):11. doi:10.14814/phy2.12699

31. Zhao Y, Santelli A, Zhu XY, et al. Low-energy shockwave treatment promotes endothelial progenitor cell homing to the stenotic pig kidney. Cell Transplant. 2020;29:963689720917342. doi:10.1177/ 0963689720917342

32. Aksoy H, Aksoy Y, Turhan H, Keles S, Ziypak T, Ozbey I. The effect of shock wave lithotripsy on nitric oxide and malondialdehyde levels in plasma and urine samples. Cell Biochem Funct. 2007;25 (5):533-536. doi:10.1002/cbf.1349

33. Park JK, Cui Y, Kim HJ, Oh HK, Koh GY, Cho KW. Activation of nitric oxide-cyclic guanosine monophosphate signaling in kidney by extracorporeal shock wave therapy. $J$ Urol. 2003;170(6 Pt 1):2459-2462. doi:10.1097/01.ju.0000094186.19728.c2
34. Lee FC, Hsi RS, Sorensen MD, et al. Renal vasoconstriction occurs early during shockwave lithotripsy in humans. J Endourol. 2015;29 (12):1392-1395. doi:10.1089/end.2015.0315

35. Griffin BR, Faubel S, Edelstein CL. Biomarkers of drug-induced kidney toxicity. Ther Drug Monit. 2019;41(2):213-226. doi:10.1097/FTD.0000000000000589

36. Yu Y, Jin H, Holder D, et al. Urinary biomarkers trefoil factor 3 and albumin enable early detection of kidney tubular injury. Nat Biotechnol. 2010;28(5):470-477. doi:10.1038/nbt.1624

37. Wadey RM, Pinches MG, Jones HB, Riccardi D, Price SA. Tissue expression and correlation of a panel of urinary biomarkers following cisplatin-induced kidney injury. Toxicol Pathol. 2014;42(3):591-602. doi:10.1177/0192623313492044

38. Lebherz-Eichinger D, Tudor B, Ankersmit HJ, et al. Trefoil factor 1 excretion is increased in early stages of chronic kidney disease. PLoS One. 2015;10(9):e0138312. doi:10.1371/journal.pone.0138312

39. Xue Y, Xu Z, Chen H, Gan W, Chong T. Low-energy shock wave preconditioning reduces renal ischemic reperfusion injury caused by renal artery occlusion. Acta Cir Bras. 2017;32(7):550-558. doi:10.1590/s0102-865020170070000006

40. Chen XJ, Zhang X, Jiang K, et al. Improved renal outcomes after revascularization of the stenotic renal artery in pigs by prior treatment with low-energy extracorporeal shockwave therapy. J Hypertens. 2019;37(10):2074-2082. doi:10.1097/HJH.0000000000002158

41. Nakamura A, Ishii A, Ohata C, Komurasaki T. Early induction of osteoactivin expression in rat renal tubular epithelial cells after unilateral ureteral obstruction. Exp Toxicol Pathol. 2007;59(1):53-59. doi:10.1016/j.etp.2007.03.005

42. Chen S, Ziyadeh FN. Vascular endothelial growth factor and diabetic nephropathy. Curr Diab Rep. 2008;8(6):470-476. doi:10.1007/ s11892-008-0081-3

43. Majumder S, Advani A. VEGF and the diabetic kidney: more than too much of a good thing. J Diabetes Complications. 2017;31 (1):273-279. doi:10.1016/j.jdiacomp.2016.10.020

44. Nakagawa T, Sato W, Kosugi T, Johnson RJ. Uncoupling of VEGF with endothelial NO as a potential mechanism for abnormal angiogenesis in the diabetic nephropathy. $J$ Diabetes Res. 2013;2013:184539. doi:10.1155/2013/184539

45. Andresdottir G, Jensen ML, Carstensen B, et al. Improved prognosis of diabetic nephropathy in type 1 diabetes. Kidney Int. 2015;87 (2):417-426. doi:10.1038/ki.2014.206

46. Andresdottir G, Jensen ML, Carstensen B, et al. Improved survival and renal prognosis of patients with type 2 diabetes and nephropathy with improved control of risk factors. Diabetes Care. 2014;37 (6):1660-1667. doi:10.2337/dc13-2036
The International Journal of Nephrology and Renovascular Disease is an international, peer-reviewed open-access journal focusing on the pathophysiology of the kidney and vascular supply. Epidemiology, screening, diagnosis, and treatment interventions are covered as well as basic science, biochemical and immunological studies. The manuscript management system is completely online and includes a very quick and fair peer-review system, which is all easy to use. Visit http://www.dovepress.com/testimonials.php to read real quotes from published authors. 